\title{
RECURSOS DE FORMULAÇÃO E VISUALIZAÇÃO DE INDICADORES PARA APOIAR PROCESSOS DE GESTÃO EDUCACIONAL EM IESS
}

Nadi Helena Presser

Eli Lopes da Silva

Raimundo Nonato Macedo dos Santos

\section{Resumo:}

Recursos de visualização das informações produzidas pelo sistema de gestão educacional da educação superior do Senac/SC, estruturado com base em indicadores de qualidade. A concepção lógica do ciclo da informação do sistema está fundamentada em uma perspectiva teórica que envolveu um diálogo multidisciplinar, buscando interfaces com autores da ciência da informação no estabelecimento de fluxos de comunicação e de estruturas de visualização da informação. Dessa forma, apresenta uma estrutura de visualização da informação interativa, utilizando a técnica conhecida como dashboard com o objetivo de auxiliar os usuários no processo de monitoramento e recuperação das informações. O trabalho enfoca como as informações produzidas pelo sistema são registradas e visualizadas com o objetivo de potencializar a apropriação da informação e ativar o conhecimento dos gestores sobre a performance das Faculdades de Tecnologia Senac em Santa Catarina.

\section{Palavras-chave:}

Indicadores; Visualização de Informação; Ciência da Informação; Gestão Educacional; Instituição de Ensino Superior

\section{RESOURCES TO FORMULATE AND TO VISUALIZE INDICATORS TO SUPPORT PROCESSES OF EDUCATIONAL MANAGEMENT IN HIGHER EDUCATION INSTITUTES}

\begin{abstract}
:
The main aims of this study is to present an information visualization resource produced for the system of educational management of higher education at Senac/SC, structuralized on the basis of quality indicators. This system is based on a theoretical perspective that involves a dialogue to multidiscipline, searching interfaces for authors of information, to establish flows of communication and visualize structures of information. It presents a structure of interactive information, using the technique known as "dashboard" with the objective of supporting the users with the monitoring process and information recovery. The work focuses on the information produced for the system to be registered and visualized with the objective of empowering the appropriate information and helping the faculty managers at Senac Technical College in Santa Catarina, Brazil.
\end{abstract}

Key-words:

Indicators; Information Visualization; Information Science

(c) Revista Digital de Biblioteconomia e Ciência da Informação,Campinas, v.7 n. 2 p. 247-259 jan./jun. 2010-ISSN: 1678-765X. 


\section{INTRODUÇÃO}

A discussão sobre gestão da educação tem sido objeto de estudo da política educacional brasileira, em diferentes momentos históricos, no cenário nacional. Trata-se de uma temática complexa, com várias perspectivas e concepções. Uma perspectiva importante implica em considerar um sistema de gestão educacional no contexto sociocultural, nas condições em que se efetiva o processo de ensino-aprendizagem, pelos aspectos relacionados ao projeto político-pedagógico, aos docentes e aos demais atores envolvidos.

O sistema de gestão de informações de qualquer natureza, quanto objeto de estudo, está compreendido no âmbito do arcabouço teórico da Ciência da Informação que abarca o estudo das propriedades gerais da informação (natureza, gênese e efeitos) e a análise dos seus processos de construção, comunicação e uso. Do ponto de vista dessa ciência, o fenômeno informação comporta um elemento de sentido, um significado em busca de um significante e, portanto, só tem validade se circula, concebida, assim, como o veículo do conhecimento. (LE CADIC, 2004).

No âmbito do seu processo de comunicação, em que gravita a compreensão do conjunto de processos sócio-culturais e econômicos, dimensões nas quais se realizam a apropriação e os usos dos produtos informacionais, envolvendo não só a produção de mensagens diferenciadas, mas também, sua distribuição adequada, as técnicas de visualização de informação, em apoio aos métodos e técnicas de tratamento e análise de informação, passaram a ser estudadas e utilizadas de forma vigorosa e recorrente a partir do início deste milênio.

Segundo Nascimento e Ferreira (2005), esses estudos levam em consideração o modo como as técnicas de visualização de informações exploram o substrato visual, as marcas e as propriedades visuais do desenho. Mostrando-se de particular interesse para os principais usuários de informação, pesquisadores, professores, gestores, as técnicas são classificadas de acordo com as características dos dados a serem visualizados.

(c) Revista Digital de Biblioteconomia e Ciência da Informação,Campinas, v.7, n. 2, p. 247-259, jan./jun. 2010- ISSN: 1678-765X. 
Entendendo ser a gestão das atividades educacionais um sistema com nível expressivo de complexidade, em contínua expansão, envolvendo decisões com sérias implicações de ordem histórica, de responsabilidade econômica; que dizem respeito ao processo de ensino aprendizagem, portanto, de conseqüências sociais, exige, como condição sine qua non, solução de continuidade e seriedade de propósito. Essas dimensões requerem processos vigorosos e robustos de acompanhamento e observação constante, e é isso o que se espera obter com as técnicas de visualização de informação.

\section{A VISUALIZAÇÃO DA INFORMAÇÃO}

A construção do sistema de gestão educacional por meio de indicadores tomou como ponto de partida o ciclo da informação tal como define Le Coadic (2004). O modelo social proposto pelo autor é de retro-alimentação, envolvendo três processos construção, comunicação e uso, que se sucedem e se alimentam reciprocamente.

O ciclo da informação ativa, de forma objetiva e dinâmica, a cadeia de produção do conhecimento. É, portanto, dinâmico e se auto-alimenta por meio de processos interativos que envolvem as necessidades de construção, comunicação e uso da informação.

Para o autor em referência, a construção compreende os processos de organizar, classificar e indexar a informação e pressupõe o envolvimento dos produtores e usuários de informações. Da mesma forma, a comunicação consiste na compreensão do conjunto de processos sócio-culturais e econômicos, contextos da apropriação do conhecimento e dos usos dos produtos informacionais, o que requer a produção de mensagens diferenciadas, a distribuição adequada, condições para assegurar o intercâmbio de informações como recurso de ativação do conhecimento para quem dele necessita.

Assim, a eficácia econômica de todo o processo do ciclo de informação proposto está referenciado e é avaliado em relação aos efeitos resultantes da apropriação da informação pelo usuário, ou seja, o uso, o consumo da informação, é o objetivo único e final de todo o processo.

(c) Revista Digital de Biblioteconomia e Ciência da Informação,Campinas, v.7, n. 2, p. 247-259, jan./jun. 2010- ISSN: 1678-765X. 
Fundado nesses conceitos e no modelo a ser apresentado, as estruturas de visualização da informação se constituem, na contemporaneidade, alternativas efetivas para o processo de compreensão e apreensão da informação,.

As técnicas de visualização buscam representar graficamente os dados de um determinado domínio de aplicação de modo que a representação visual gerada explore a capacidade de percepção do homem e este, a partir das relações espaciais exibidas, interprete e compreenda as informações apresentadas e, finalmente deduza novos conhecimentos.” (FREITAS et al, 2001, p. 144)

Autores em várias áreas reconhecem a importância dos recursos de visualização da informação. Card et al (1999) e Chen (2002) apontam as técnicas de visualização de informação como recursos que otimizam o uso das capacidades visuais humanas para compreender fenômenos que não possuem em si mesmos uma representação espacial própria. A visualização da informação, segundo Card et al, procura reunir ambos, o poder da percepção visual humana e o poder de processamento do computador para analisar e compreender um conjunto de informações. Estivalet (2000) afirma que um dos aspectos da visualização da informação na otimização da cognição humana está no fato de ser mais fácil para uma pessoa compreender o significado de uma imagem do que o significado de vários dados isolados. Oliveira (2007) aponta a facilidade de construir conclusões e detectar padrões a partir de uma representação visual. Chen (2006) considera a visualização da informação como um recurso que auxilia as pessoas a encontrarem a informação de que precisam intuitivamente e de maneira mais efetiva.

Entre as técnicas de visualização da informação consagradas, o dashboard pode ser entendido como "painel de controle”, “indicador de bordo" ou simplesmente "painel de indicadores”. Normalmente, o produto final é composto de uma ou mais páginas na web com informações de mais alto nível gerencial, coletados por meio de dados dispostos em banco de dados, em planilhas eletrônicas ou outras fontes de informação.

Os dashboards reúnem um conjunto de estruturas de visualização de informações de modo que a interação entre elas fornece significado ao exibirem suas relações, permitem visualizar informações do geral para o detalhe (DIAS, 2007).

A capacidade de ramificação, segundo esse autor, é um aspecto muito importante dos dashboard. A manipulação de cada estrutura presente no painel causa alterações nas 
demais, provocando uma alteração de comportamento no painel, possibilitando a visualização de informações de forma interativa. E, conforme o usuário interage com as partes do painel, esse deve apresentar, em proporção maior, os detalhes sobre as informações solicitadas, técnica conhecida como overview-detail.

\section{A CONSTRUÇÃO DO DASHBOARD DE INDICADORES DE GESTÃO EDUCACIONAL}

\subsection{O processo de construção dos indicadores}

A principal característica de um indicador é a sua capacidade de sintetizar um conjunto de informações, representando apenas o significado essencial dos aspectos analisados. Segundo Bellen (2005), os indicadores agregam e quantificam informações de modo que sua significância torne-se mais evidente.

Definir o processo de construção dos indicadores exigiu, inicialmente, uma compreensão da lógica do sistema da educação superior do Senac em Santa Catarina: como ele funciona e opera no contexto da educação profissional e tecnológica para, posteriormente, identificar as informações a serem produzidas pelo sistema e como estruturá-las, de forma a garantir melhores mecanismos de busca e recuperação e melhores resultados no processo de seu uso.

Assim, consideraram-se indicadores que representam todos os aspectos do sistema, aptos para medir o desempenho tanto em âmbito local das sete faculdades como estadual. Nessas condições, o sistema contempla o conjunto de todas elas nos aspectos relativos aos fins e às especificidades próprias do ensino superior do Senac/SC e da sua lógica de funcionamento. Nessas reflexões, foram consideradas questões referentes aos docentes; aos pressupostos filosóficos teórico-metodológicos definidos no projeto político institucional; à cultura organizacional; ao papel basilar das políticas de regulação da educação superior; aos projetos pedagógicos dos cursos e à indissociabilidade entre ensino, pesquisa e extensão como atividades interdependentes que precisam assegurar ênfase equivalente na educação profissional e tecnológica do Senac/SC.

(c) Revista Digital de Biblioteconomia e Ciência da Informação,Campinas, v.7, n. 2, p. 247-259, jan./jun. 2010- ISSN: 1678-765X. 
Essas considerações possibilitaram organizar os indicadores em categorias relevantes à sua tarefa de análise, o que permite consultá-los e compará-los com relação às suas características e às suas especificidades, como mostra o Quadro 1, o que pode oferecer uma visão ampla e exaustiva do sistema educacional.

\begin{tabular}{|cl|}
\hline \multicolumn{2}{|l|}{ Categorias de Indicadores } \\
\hline 1. & Docentes \\
\hline 2. & Adoção dos princípios filosóficos teórico-metodológicos \\
\hline 3. & Alunos \\
\hline 4. & Trabalho de Conclusão de Último Semestre - TCS \\
\hline 5. & Atividade Complementar de Caráter Interdisciplinar \\
\hline 6. & Pesquisa e Extensão \\
\hline 7. & Egressos \\
\hline 8. & Matrículas \\
\hline 9. & Avaliação Externa \\
\hline 10. & Auto-Avaliação Institucional \\
\hline & Quadro 1: Categorias de Indicadores \\
\hline
\end{tabular}

Observa-se que, cada um das categorias apresentadas é um ângulo de análise do desempenho das Faculdades e o seu conjunto permite a visualização da performance em todas as dimensões julgadas importantes, sintetizando as informações essenciais do sistema.

No Quadro 2, constam os indicadores definidos segundo a lógica do sistema de educação superior do Senac em Santa Catarina. Por outro lado, cada indicador foi construído de forma a comportar características que exprimissem a sua totalidade. 


\begin{tabular}{|c|c|c|c|}
\hline \multicolumn{4}{|c|}{ Categoria: Docentes } \\
\hline Critério & Indicador & $\begin{array}{l}\text { Valores de } \\
\text { Referência }\end{array}$ & $\begin{array}{l}\text { Padronização da Coleta } \\
\text { das Informações }\end{array}$ \\
\hline Avaliação docente & $\begin{array}{l}\text { \% de docentes } \\
\text { avaliados com } \\
\text { conceitos: } \\
\text { a. Ótimo; } \\
\text { b. Bom; } \\
\text { c. Satisfatório; } \\
\text { d. Insatisfatório. }\end{array}$ & $\begin{array}{l}80 \% \text { dos docentes } \\
\text { com avaliação } \\
\text { mínima no } \\
\text { somatório de } \\
\text { conceitos Bom e } \\
\text { Ótimo. }\end{array}$ & $\begin{array}{l}\text { Fonte: relatório da pesquisa } \\
\text { online de Avaliação } \\
\text { Docente. } \\
\text { População: considerar } \\
\text { mínimo de } 70 \% \text { de alunos } \\
\text { respondentes, em cada } \\
\text { turma, de cada curso. } \\
\text { Responsável: a pesquisa } \\
\text { deve ser realizada pelo } \\
\text { coordenador do curso, após } \\
40 \text { dias letivos, antes do } \\
\text { Conselho de Classe } \\
\text { Intermediário. } \\
\text { Periodicidade: semestral. }\end{array}$ \\
\hline Titulação & $\begin{array}{l}\text { \% de docentes com } \\
\text { titulação: } \\
\text { e. Doutor; } \\
\text { f. Mestre; } \\
\text { g. Especialista. }\end{array}$ & $\begin{array}{l}60 \% \text { dos docentes } \\
\text { de cada curso com } \\
\text { titulação obtida em } \\
\text { programas de pós- } \\
\text { graduação stricto } \\
\text { sensu e, destes, } \\
50 \% \text { doutores. }\end{array}$ & $\begin{array}{l}\text { Fonte: Projetos } \\
\text { Pedagógicos dos Cursos } \\
\text { (PPCs) específicos de cada } \\
\text { curso/Faculdade, } \\
\text { atualizados até a data de } \\
\text { início das aulas em cada } \\
\text { semestre. } \\
\text { População: considerar } \\
\text { 100\% dos docentes de cada } \\
\text { curso. } \\
\text { Responsável: Mantenedora. } \\
\text { Periodicidade: semestral. }\end{array}$ \\
\hline
\end{tabular}

Quadro 2: Composição dos indicadores da categoria docentes

Vale chamar a atenção que: critérios são compreendidos como a essência daquilo que se quer avaliar e que se julga poder esperar legitimamente do processo. Assim, os indicadores descrevem de forma judiciosa em que parâmetros e o que avaliar em cada critério, tornando o processo avaliativo transparente e facilitando o diálogo entre mantenedora e mantida.

Nessa etapa, para permitir a descrição judiciosa dos indicadores, foram realizadas análises dos aspectos relacionados à relevância, gradação de intensidade, univocidade, padronização e rastreabilidade dos indicadores, segundo preconiza Trzesniak (1998). Assim, cada indicador exprime um aspecto ou uma característica específica da instituição 
que pode ser mensurada. São considerados imprescindíveis os indicadores que representam condições obrigatórias definidas pela legislação para o acesso e a permanência do Senac/SC no sistema de ensino superior. Entre as categorias apresentadas no Quadro 1 foi estabelecido um total de 60 (sessenta) indicadores.

Ainda com relação ao Quadro 2, os valores de referência estabelecem níveis de qualidade coerentes com a política da educação profissional e tecnológica do Senac/SC, do Ministério da Educação e Cultura (MEC) e do Sistema Nacional de Avaliação da Educação Superior (SINAES), tornando-se metas a alcançar. A atribuição de valores de referência para cada um dos indicadores se constitui em um importante foco de intervenção dos atores educacionais envolvidos no processo de avaliação. Por outro lado, a padronização da coleta das informações especifica o local em que os dados podem ser coletados, a metodologia de sua obtenção, bem como o período de coleta, de forma a tornar o processo bem definido e estável.

\subsection{O processo de comunicação dos indicadores}

O processo de comunicação dos indicadores consistiu em identificar estruturas de visualização da informação, assegurando recursos gráficos e formatos em que a informação pudesse ser visualizada de forma simplificada, porém interativa, em vários níveis de detalhe, utilizando imagens, figuras e gráficos de modo a reduzir o tempo gasto pelos usuários para recuperar as informações necessárias.

As técnicas de visualização de informação são compreendidas no processo de gestão educacional como um recurso interativo de representação de informações com o objetivo de compreender o comportamento de cada indicador no processo, permitindo obter maior clareza e precisão sobre a situação estudada.

Para a visualização e análise dos resultados junto às instâncias de competência, em tempo real e de forma simples, optou-se por representar os indicadores do sistema de gestão educacional utilizando a técnica dashboard.

Denominado "Dashboard de Indicadores de Gestão Educacional", a técnica vincula toda a estrutura de informação da educação superior do Senac/SC a um sistema de 
indicadores, de tal forma a apresentar uma visão geral do sistema e a interação entre as variáveis que operam no seu interior de modo a identificar problemas de gestão gerais e específicos.

\subsection{Construção do Dashboard de Indicadores de Gestão Educacional}

A utilização da técnica do dashboard permite que o processo da coleta e registro dos dados torne-se simples, econômico e, sobretudo de fácil manuseio pelos usuários, além de agregar característica de interoperabilidade, permitindo vínculos entre as planilhas eletrônicas de cada Faculdade com o Banco de Dados da mantenedora.

Sempre que há alteração nas planilhas das Faculdades, os vínculos com o Banco de Dados da mantenedora são atualizados e exportados novamente para as planilhas que geram o dashboard publicado na intranet.

\subsubsection{Visualização dos indicadores}

As páginas são disponibilizadas por categoria conforme mostra a Figura 1. Assim, foi criada uma página para cada categoria apresentada no Quadro 1. A página web “docentes.htm” tem o formato apresentado na Figura 1, visualizada através do navegador (browser) de internet, que pode ser o Mozilla, Internet Explorer® ou outro. 


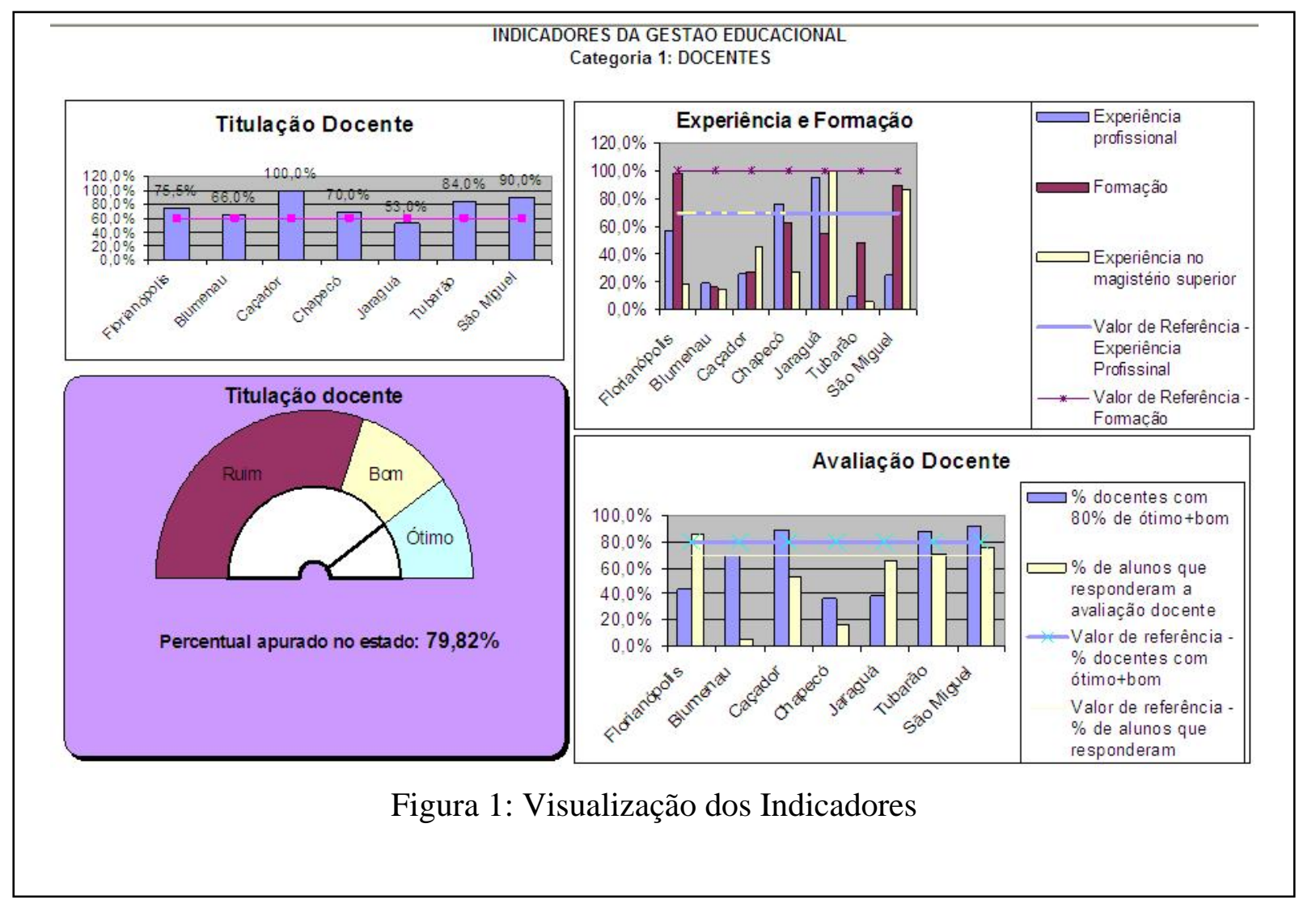

No caso dos gráficos de barra, por exemplo, tem-se uma linha horizontal que representa os valores de referência. Para o gráfico de barras “Titulação Docente”, o primeiro à esquerda, na Figura 1, o valor de referência de 60\% é igual para todas as Faculdades, sendo representado por uma linha em cor diferente das barras. Entretanto, para o gráfico Experiência Docente”, localizado à direita e no alto da Figura 1, existem 3 (três) linhas de valor de referência, sendo que cada linha representa o valor de referência do indicador que possui uma barra de mesma cor. A barra azul (Experiência Profissional) tem seu valor de referência representado por uma linha azul, a barra vermelha (Formação) tem o seu valor de referência representado por uma linha vermelha e assim por diante.

\subsubsection{Vinculação dos dados}

Na mantenedora, os dados são registrados em um Banco de Dados. Na figura 2 tem-se uma representação de um dos formulários utilizados para visualização e armazenamento dos dados da avaliação docente. Esses dados são exportados em formato de planilhas eletrônicas, contendo o total dos indicadores apurados e vinculadas com as planilhas de cada Faculdade. 


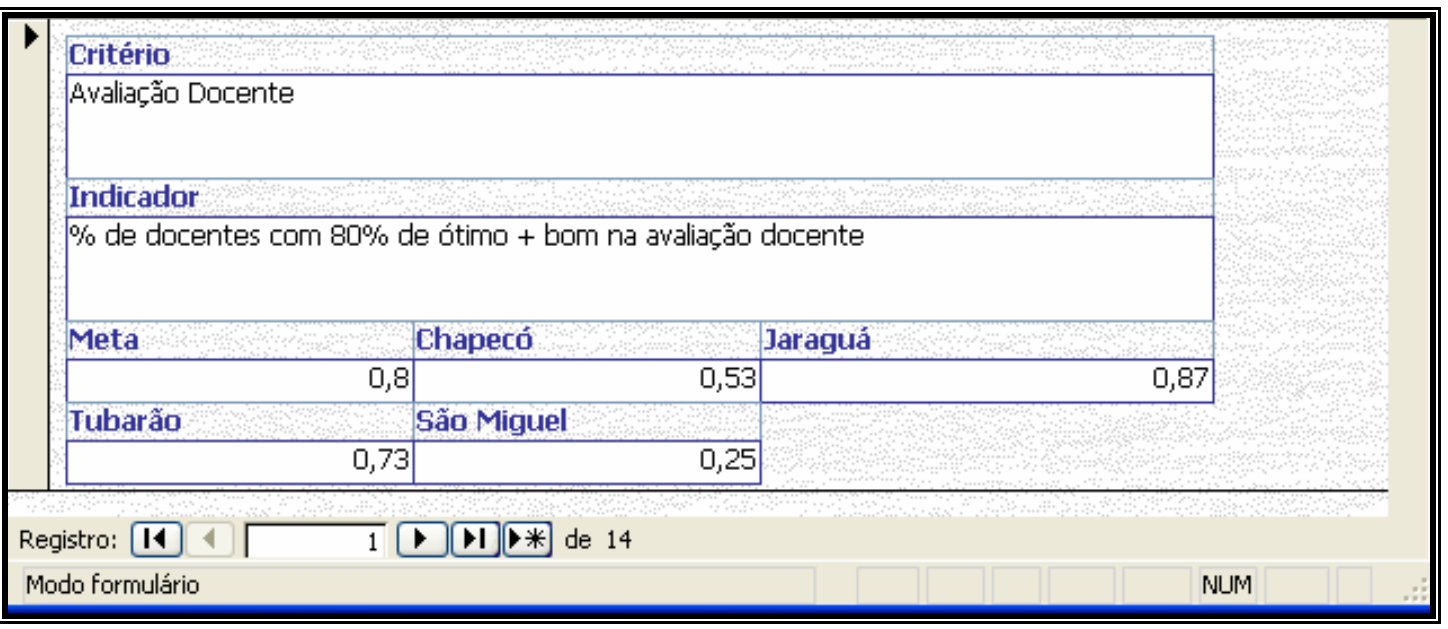

Figura 2: Formulário gerado a partir do banco de dados da mantenedora

A Figura 3 ilustra a forma como os dados são coletados em cada Faculdade, para posterior migração para o Banco de Dados da mantenedora.

\begin{tabular}{|c|c|c|c|c|}
\hline \multicolumn{2}{|r|}{ D5 } & \multirow[b]{2}{*}{$\mathrm{B}$} & \multirow[b]{2}{*}{$\mathrm{C}$} & \multirow[b]{2}{*}{$\mathrm{D}$} \\
\hline & A & & & \\
\hline 1 & Critério & Indicador & Meta & Chapecó \\
\hline 2 & Avaliaçẫo Docente & $\begin{array}{l}\% \text { de docentes com } 80 \% \text { de ótimo }+ \text { bom na } \\
\text { avaliaçẫo docente }\end{array}$ & $80 \%$ & $53 \%$ \\
\hline 3 & Avaliaçẫo Docente & $\begin{array}{l}\% \text { de docentes com } 2 \text { a. avaliaçẫo abaixo da meta e } \\
\text { que permanecem no quadro }\end{array}$ & $0 \%$ & $0 \%$ \\
\hline 4 & Avaliaçẫo Docente & $\%$ de alunos que responderam a avaliação docente & $70 \%$ & $86 \%$ \\
\hline 5 & $\begin{array}{l}\text { Acompanhamento } \\
\text { pedagógico da atividade } \\
\text { docente }\end{array}$ & $\begin{array}{l}\text { Número de docentes, por curso, avaliados pelo } \\
\text { pedagogo, em sua prática docente }\end{array}$ & $100 \%$ & \\
\hline & - M $\backslash$ Gráficos $入$ GP-Do & centes / GP-Princípios / GP-Aluno /GP-TCS / GP-. & & $>$ \\
\hline
\end{tabular}

Figura 3: Planilha do arquivo disponibilizado para a Faculdade

A opção da mantenedora em armazenar os dados em Banco de Dados vem da necessidade e também da facilidade de gerar relatórios das informações contidas no banco de dados. Assim, além do Dashboard de Indicadores de Gestão Educacional que apresenta as informações em um nível macro, destinado aos gerentes de posições mais estratégias, é possível gerar relatórios para gestores de nível intermediário, que necessitam da informação com um grau maior de detalhamento. 


\subsubsection{Atualização das informações}

A atualização das informações é realizada de baixo para cima, ou seja, partindo-se dos detalhes para que os vínculos entre as planilhas e entre os dashboards atualizem automaticamente o nível mais macro. Dessa forma, quando a mantenedora recebe as planilhas eletrônicas atualizadas pelas Faculdades, exporta os dados dessas planilhas para o Banco de Dados.

A partir do momento em que o Banco de Dados é atualizado, são geradas as planilhas que fazem ligação com o dashboard, disponível na intranet (Figura 1), tornando a informação do nível mais macro fidedígna aos dados fornecidos na ponta do processo.

\section{CONCLUSÃO}

O conjunto de soluções apresentados não esgota as possibilidades de visualização das informações produzidas pelo sistema de gestão educacional, mas indica caminhos pelos quais o sistema pode evoluir.

O "Dashboard de Indicadores de Gestão Educacional" é a apresentação visual das dez categorias de indicadores, sintetizando o estado geral do sistema educacional, o que contribui para aumentar o conhecimento do processo da educação superior em uma abordagem ampla e exaustiva, orientando a tomada de decisão em medidas específicas e gerais de ação.

Por outro lado, o sistema de gestão construído se constitui em um registro da série histórica da memória coletiva (HALBWACHS, 1990) da performance do Senac/SC, no contexto da educação superior, possibilitando ao mesmo tempo avaliar os usos dados à informação e fornecer elementos para avaliar os efeitos resultantes desses usos nas atividades dos usuários (LE COADIC, 2004).

\section{REFERÊNCIAS}

BELLEN, H.M.V. Indicadores de sustentabilidade. Uma análise comparativa. Rio de Janeiroo:Editora FGV, 2005. CARD, S. K.; MACKINLAY, J. D.; SHNEIDERMAN, B., Readings in information visualization using vision to think, San Francisco, 1999. 
CHEN, C. Information Visualization: beyond the horizon. Springer, 2006.

DIAS, M. P. A contribuição da Visualização da Informação para a Ciência da

Informação. 2007. Dissertação (Mestrado em Ciência da Informação), Pontifícia Universidade Católica de Campinas - PUC-Campinas, 2007.

ESTIVALET, L. F. O Uso de Ícones na visualização de Informações. Dissertação Computação, UFRS, Porto Alegre, 2000.

FREITAS, C. M.D.S.; et al. Introdução à visualização de Informações. Revista de Informática Teórica e Aplicada, v.8, n. 2, 2001.

HALBWACHS, M. A memória coletiva. São Paulo: Vértice, 1990.

LE COADIC, Yves-François. A Ciência da Informação. Tradução de Maria Yêda F.S. de Filgueiras Gomes. 2.ed.. Brasília, DF: Briquet de Lemos, 2004.

NASCIMENTO, Hugo A. D.; FERREIRA, Cristiane, B. R. Visualização de informações: uma abordagem prática. In: CONGRESSO DA SOCIEDADE BRASILEIRA DE COMPUTAÇÃO, 25, 2005 Anais... São Leopoldo: UNISINOS, 2005.

TRZESNIAK, P. Indicadores quantitativos: reflexões que antecedem seu estabelecimento. Ciência da Informação. Brasília, v.27, n. 2, p. 159-164, maio/ago. 1998.

Nadi Helena Presser

Professora Doutora, coordena a Educação Superior do SENAC SC. nadipresser@terra.com.br

\section{Eli Lopes da Silva}

Professor Mestre, atua junto à Coordenação de Ensino Superior do SENAC SC e docente nos cursos superiores de tecnologia do SENAI SC. elisilva@sc.senac.br

\section{Raimundo Nonato Macedo dos Santos}

Professor Doutor, Programa de Pós-Graduação em Ciência da Informação do Departamento de Ciência da Informação da Universidade Federal de Santa Catarina. raimundo@cin.ufsc.br

Recebido em: 13/03/2009

Aceito para publicação em: jul/2009

(c) Revista Digital de Biblioteconomia e Ciência da Informação,Campinas, v.7, n. 2, p. 247-259, jan.jjun. 2010- ISSN: 1678-765X. 